\title{
Índice de Massa Corporal, Circunferência da Cintura e Dobra Cutânea Triciptal na Predição de Alterações Lipídicas em Crianças com 11 Anos de Idade
}

\begin{abstract}
RESUMO
Objetivo: Analisar a capacidade preditiva do índice de massa corporal (IMC), da circunferência da cintura (Ccin) e da dobra cutânea triciptal (TR) no diagnóstico de alterações lipídicas e sugerir pontos de corte para tais medidas na identificação da doença em crianças. Metodologia: Avaliou-se o IMC, a Ccin, a TR e os níveis lipídicos de 374 escolares $(11,25 \pm 0,28$ anos $)$ da rede de ensino da cidade de Santa Maria, RS. A análise estatística foi realizada por meio da curva ROC (IC95\%), da sensibilidade, da especificidade e dos valores preditivos. Resultados: As variáveis antropométricas apresentaram capacidade diagnóstica apenas para TG no sexo feminino, CT e LCL-C no sexo masculino. Conclusão: Conclui-se que o IMC correspondente a $19,3 \mathrm{~kg} / \mathrm{m}^{2}$ é um valor diagnóstico para alterações no TG, no sexo feminino, e para CT e LDL-C, no sexo masculino. As concentrações elevadas de CT e LDL-C, no sexo masculino, também podem ser diagnosticadas a partir de valores superiores a $65,7 \mathrm{~cm}$ para Ccin e $21 \mathrm{~mm}$ para TR. (Arq Bras Endocrinol Metab 2008; 52/6:1009-1014)
\end{abstract}

Descritores: Estudantes; Circunferência da cintura; Tríceps; Índice de massa corporal; Colesterol

\begin{abstract}
Body Mass Index, Waist Circumference and Skinfolds for Predicting Lipid Abnormalities in 11 Years Old Children.

Objective: To analyze the predictive capacity of Body Mass Index (BMI), Waist Circumference (WC) and Triceps Skin Folds (TSF) to diagnose of lipid abnormalities and to suggest cutoff points for these measures in order to identify this disease in children. Methodology: It was measured the BMI, WC, TSF and lipid profiles of 374 schoolchildren $(11,25 \pm 0,28$ years $)$ from the public education system of Santa Maria, RS, Brazil. Area under the ROC curve $(95 \% \mathrm{Cl})$, sensitivity, specificity and predictive values were analyzed in order to identify the ideal cutoff points. Results: The anthropometric variables only exhibited diagnostic capacity for TG among the girls and CT and LDL-C for the boys. Conclusions: It was concluded that a BMI of $19.3 \mathrm{~kg} / \mathrm{m}^{2}$ is a diagnostic value for abnormal TG levels in females and for abnormal CT and LDL-C among males. Elevated concentrations of CT and LDL-C can also be diagnosed in boys using cut-off values of WC $>65.7 \mathrm{~cm}$ and TSF $>21.0 \mathrm{~mm}$. (Arq Bras Endocrinol Metab 2008; 52/6:1009-1014)
\end{abstract}

Keywords: Students; Waist circumference; Triceps; Body mass index; Cholesterol

\section{INTRODUÇÃo}

DISLIPIDEMIA, CONDIÇÃO NA qual há alterações metabólicas lipídicas, ocasionando aumento (ou diminuição no caso da lipoproteína de alta densidade, o HDL-C) na concentração sérica das lipoproteínas (1), atinge cerca de 17,6\% original

\author{
CláUdia Cruz Lunardi \\ Édo LuIz PetroskI
}

Núcleo de Cineantropometria e Desempenho Humano da Universidade Federal de Santa Catarina (UFSC), Florianópolis, SC, Brasil.
Recebido em 13/5/2008 Aceito em 3/7/2008 
da população infantil de Portugal (2), 27,98\% de Bento Gonçalves, RS (3) e 35\% de Campinas, SP (4).

Os indivíduos dislipidêmicos possuem freqüentemente obesidade central $(5,6)$ que, em excesso, promove a liberação de ácidos graxos livres e triglicerídeos (TG) na circulação (7). Em vista disso, autores sugerem a possibilidade de empregar uma ou mais medidas antropométricas de adiposidade central como o primeiro passo para projetar dislipidemias $(5,6)$.

A circunferência da cintura (Ccin) é uma ferramenta importante para verificar sobrepeso e obesidade em crianças, identificando, assim, aquelas que apresentam risco ao desenvolvimento de complicações metabólicas e cardiovasculares (8). Em razão de sua facilidade de medida e também por apresentar associação consistente, e geralmente forte, com concentrações alteradas para fatores de risco [concentrações anormais de lipoproteína de baixa densidade (LDL-C), HDL-C e TG e insulina], é apropriada para estudos epidemiológicos em crianças (9). No entanto, as diferenças na composição corporal (marcantes mudanças nas circunferências, dobras cutâneas) dos diversos grupos etários e raciais e as alterações nas concentrações de lipoproteínas durante o crescimento e desenvolvimento dificultam o emprego de pontos de corte universais para a medida $(9,10)$.

Assim como a Ccin, o índice de massa corporal (IMC) e as dobras cutâneas (em especial o tríceps) são largamente utilizados no diagnóstico de indivíduos com excesso de peso, por causa de sua praticidade e do baixo custo. O IMC apresenta forte associação com o aumento do risco de desenvolver doenças cardiovasculares, hipertensão $(11,12)$, concentrações elevadas de LDL-C e TG no sangue e diabetes $(11,13)$. A medida das dobras cutâneas vem sendo utilizada em estudos populacionais para a avaliação do tecido adiposo subcutâneo, principalmente para identificar os pacientes com excesso de gordura.

Diante do exposto, este estudo tem como objetivo analisar a capacidade preditiva das medidas antropométricas (IMC, Ccin e TR) no diagnóstico de alterações lipídicas e sugerir pontos de corte para tais medidas na identificação de dislipidemias em escolares.

\section{MÉTODOS}

Este estudo caracteriza-se como de corte transversal, realizado no período de agosto a dezembro de 2005 , no município de Santa Maria, RS. O tamanho da amos- tra foi calculado com grau de confiança de 95\%, margem de erro de $5 \%$, estratificados por rede de ensino (municipal, estadual, federal e particular) e sexo (352 escolares). Como critérios de inclusão para o estudo, exigiu-se que os indivíduos fossem nascidos no ano de 1994, sendo excluídos os estudantes que não respeitaram jejum de 12 horas para os exames laboratoriais. $\mathrm{O}$ estudo foi apresentado nas escolas sorteadas, para todos os responsáveis, por meio de carta que continha os objetivos do projeto e as explicações referentes a lipídios séricos. Avaliaram-se todos os estudantes que demonstraram interesse, totalizando 374 (208 meninas e 166 meninos), com idade média de 11,25 $\pm 0,28$ anos.

Os escolares que apresentaram alterações lipídicas receberam orientações quanto à importância do controle sérico e dos benefícios da alimentação saudável e das atividades físicas.

O Laboratório de Análises Clínicas da Universidade Federal de Santa Maria (LAC-UFSM) ficou responsável pela coleta e análise sangüínea dos dados. Uma técnica laboratorial deslocou-se às escolas e coletou $4 \mathrm{ml}$ de sangue por punção venosa a vácuo de cada indivíduo, após jejum de 12 horas, em frascos secos para dosagens bioquímicas, no horário compreendido entre $7 \mathrm{~h} 30 \mathrm{~min}$ e $10 \mathrm{~h}$. As análises sangüíneas foram realizadas pelo método colorimétrico-enzimático para todas as dosagens (CT, HDL-C e TG), utilizando o aparelho 917 Automatic Analyser Hitachi (Boehringer Mannhein). O LDL-C foi calculado pela fórmula: [(CT HDL-C)-(TG/5)], que somente é válida quando o valor do TG for inferior a $400 \mathrm{mg} / \mathrm{dL}$ (14). Todas as crianças deste estudo adequaram-se à fórmula (apresentaram valores para $\mathrm{TG}$ inferior a $400 \mathrm{mg} / \mathrm{dL}$ ).

As avaliações antropométricas seguiram os critérios padronizados, quais sejam, estatura (EST), massa corporal (MC) (15), Ccin (16) e dobra cutânea do tríceps (TR) (17). As mensurações foram realizadas por apenas um avaliador que utilizou fita métrica para medidas antropométricas (escala de $0,1 \mathrm{~mm}$ ), balança marca Plena, estadiômetro de metal Cardiomed e plicômetro da marca Cescorf para mensuração da massa corporal, estatura e dobra cutânea, respectivamente. A partir dos escores de MC e EST, foi calculado o IMC, de acordo com a equação: $\operatorname{IMC~}\left(\mathrm{kg} / \mathrm{m}^{2}\right)=\mathrm{MC}(\mathrm{kg}) / \mathrm{EST}^{2}(\mathrm{~m})$.

Utilizou-se a classificação proposta pela III Diretrizes Brasileiras sobre Dislipidemias (1) como valores de referência para este estudo, que considera valores aumentados para CT $\geq 200 \mathrm{mg} / \mathrm{dL}$, HDL-C $<35 \mathrm{mg} /$ $\mathrm{dL}, \mathrm{LDL}-\mathrm{C} \geq 130 \mathrm{mg} / \mathrm{dL}$ e para $\mathrm{TG}>130 \mathrm{mg} / \mathrm{dL}$. 
Tabela 1. Dados descritivos das variáveis antropométricas e bioquímicas da amostra.

\begin{tabular}{|c|c|c|c|c|c|}
\hline \multicolumn{6}{|c|}{ Variáveis antropométricas } \\
\hline & $\mathrm{n}$ & IMC & Ccin & TR & \\
\hline Feminino & 208 & $18,5 \pm 3,1$ & $62,3 \pm 6,7$ & $16,3 \pm 7,1^{*}$ & \\
\hline Masculino & 166 & $19,0 \pm 3,6$ & $64,1 \pm 8,4$ & $15,1 \pm 8,3$ & \\
\hline \multicolumn{6}{|c|}{ Variáveis bioquímicas } \\
\hline & $\mathrm{n}$ & CT & HDL-C & LDL-C & TG \\
\hline Feminino & 208 & $154,4 \pm 26,1$ & $49,2 \pm 10,9$ & $88,0 \pm 22,8$ & $85,8 \pm 43,3^{*}$ \\
\hline Masculino & 166 & $151,7 \pm 24,8$ & $50,2 \pm 10,0$ & $87,3 \pm 21,1$ & $71,0 \pm 33,4$ \\
\hline
\end{tabular}

${ }^{*} p<0,05$.

Para identificar possíveis diferenças entre os sexos, empregou-se o teste $t$ de Student para amostras independentes, uma vez que foi detectada normalidade nos dados (teste de Kolgomorov-Smirnov). Foram utilizados a análise da curva ROC (receiver operator characteristic curve) $\mathrm{e}$ o intervalo de confiança (IC95\%) para detectar as variáveis antropométricas que apresentaram capacidade preditiva; e análise de sensibilidade, especificidade e valores preditivos para identificação dos pontos de corte ideais para as medidas antropométricas como marcadores de dislipidemia. A análise da área sobre a curva ROC parte do pressuposto que quanto maior a área, maior o poder discriminatório da medida antropométrica para a variável bioquímica. $\mathrm{O}$ IC determina que a capacidade preditiva do indicador de obesidade não acontece em virtude do acaso e o seu limite inferior não deve ser menor do que 0,50 (18).

Recorreu-se aos valores preditivos com o intuito de analisar a probabilidade de doença, dado os resultados do teste diagnóstico. Assim, o valor preditivo positivo (VPP) expressa a possibilidade de um paciente com o teste positivo ter a doença e o valor preditivo negativo (VPN) notifica a probabilidade de um indivíduo com o teste negativo não ter a doença. Para a estimativa de ambos, utilizaram-se as seguintes equações (19):

$$
\begin{aligned}
& \mathrm{VPP}=\frac{(\text { Sens } \times \text { Prev })}{(\text { Sens } \times \text { Prev })+(1-\text { Espec }) \times(1-\text { Prev })} \\
& \mathrm{VPN}=\frac{\text { Espec } \times(1-\text { Prev })}{(1-\text { Sens }) \times \text { Prev }+ \text { Espec } \times(1-\text { Prev })}
\end{aligned}
$$

em que: Sens = sensibilidade; Espec $=$ especificidade $; \mathrm{e}$ Prev $=$ prevalência da doença.

As análises foram realizadas no pacote estatístico SPSS for Windows versão 14.0, considerando o intervalo de confiança de $95 \%$.

O estudo foi aprovado pelo Comitê de Ética em Pesquisa da Universidade Federal de Santa Maria
(CAAE 0018.0.243.000-05). Para participar das coletas, os pais, ou responsáveis, assinaram o termo de consentimento livre e esclarecido (TCLE).

\section{RESULTADOS}

As características descritivas das variáveis antropométricas e bioquímicas do estudo estão apresentadas na Tabela 1. Avaliaram-se 208 escolares do sexo feminino (56\%) e 166 do sexo masculino (44\%), com idade média de $11,24 \pm 0,27$ anos e $11,26 \pm 0,30$ anos, respectivamente, os quais relataram diferença estatística significante superior para o sexo feminino nas variáveis TR e TG.

A prevalência de alterações lipídicas variou de 3,6\% a $10,1 \%$, não sendo encontrada diferença estatística significante entre os gêneros (Figura 1).

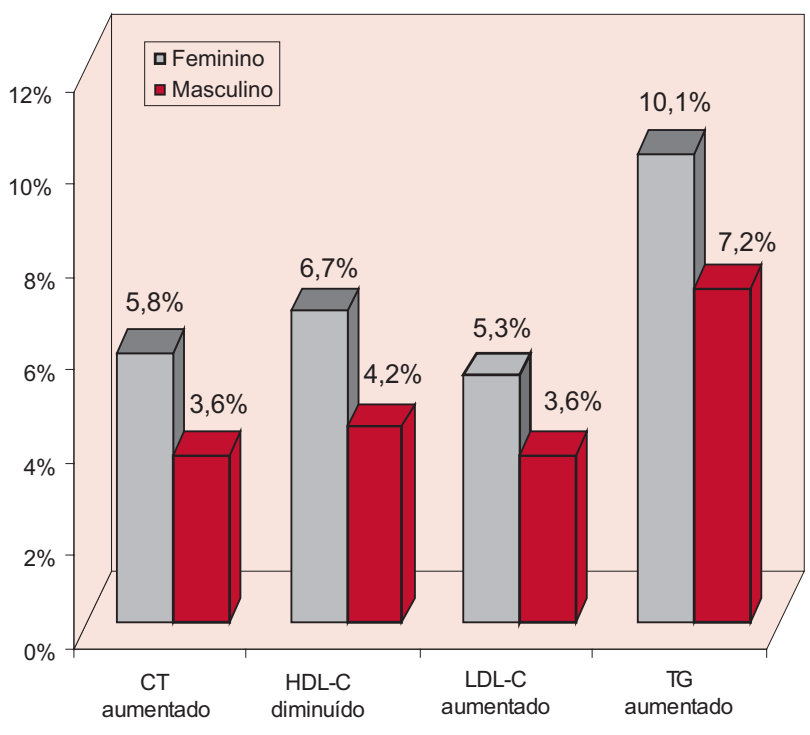

Figura 1. Prevalência de alterações bioquímicas. 
As Tabelas 2 e 3 apresentam somente as áreas sob a curva ROC das variáveis antropométricas que se mostraram diagnósticas para alterações lipídicas nos sexos feminino e masculino, respectivamente. Apresentamse, também, os pontos de corte que relataram melhor equilíbrio entre sensibilidade e especificidade, inclusive o percentil em que se encontram e os seus valores preditivos. Verificou-se variação nas áreas sob a curva ROC de 0,62 a 0,87 . A sensibilidade e a especificidade e os valores preditivos apresentaram amplitude de $50 \%$ a $100 \%$ e $8,7 \%$ a $100 \%$, respectivamente.

\section{DISCUSSÃO}

Este estudo, inicialmente, analisou a capacidade preditiva do IMC, da Ccin e das TR no diagnóstico de alterações lipídicas em crianças com idade média de 11,25 $\pm 0,28$ anos, para, posteriormente, sugerir pontos de corte, visando a identificar concentrações indesejáveis de lipídios séricos.
As prevalências de alterações lipídicas não diferiram entre os sexos e revelaram baixa ocorrência de dislipidemia na amostra estudada, sendo essas prevalências inferiores às relatadas em outras localidades $(2-4,20,21)$.

Ao serem analisadas as áreas sob as curvas ROC, resultantes de cada variável antropométrica para cada variável bioquímica, consideraram-se válidas apenas oito das 30 áreas propostas (Tabelas 2 e 3 ). Os VPP encontrados foram baixos, fato este explicado pela forte dependência dessa medida da prevalência de dislipidemia, mesmo se a sensibilidade e a especificidade do teste fossem elevadas. Os VPN indicaram que há grande possibilidade de indivíduos considerados com níveis lipídicos desejáveis estarem realmente sadios $(19,22)$.

\section{IMC na predição de dislipidemia}

O IMC mostrou-se preditor, no sexo feminino, para TG e no sexo masculino, para CT e LDL-C (limite inferior do IC superior a $60 \%$ ). Coincidentemente, o valor de IMC de $19,3 \mathrm{~m}^{2}(\mathrm{z}=0,08$ para sexo masculino e $\mathrm{z}=0,25$ para

Tabela 2. Áreas sob a curva ROC, pontos de corte propostos e respectivos valores preditivos para variáveis antropométricas e bioquímicas do sexo feminino.

\begin{tabular}{|c|c|c|c|c|c|c|c|c|}
\hline & Bioquímica & Área (IC95\%) & Pto & $\mathbf{P}$ & Sens & Espec & VPP & VPN \\
\hline IMC & TG & $\begin{array}{c}0,74 \\
(0,63-0,86)\end{array}$ & 19,3 & 68 & 71,4 & 73,3 & 22,9 & 95,8 \\
\hline Ccin & TG & $\begin{array}{c}0,74 \\
(0,62-0,87)\end{array}$ & 65,1 & 77 & 62,0 & 81,3 & 26,9 & 95,0 \\
\hline
\end{tabular}

Área $(\mathrm{IC95 \% )} \mathrm{=} \mathrm{área} \mathrm{sob} \mathrm{a} \mathrm{curva} \mathrm{ROC} \mathrm{e} \mathrm{intervalo} \mathrm{de} \mathrm{confiança;} \mathrm{Pto} \mathrm{=} \mathrm{ponto} \mathrm{de} \mathrm{corte} \mathrm{proposto;} \mathrm{P} \mathrm{=} \mathrm{percentil} \mathrm{em} \mathrm{que} \mathrm{se} \mathrm{encontra;} \mathrm{Pto;} \mathrm{Sens} \mathrm{=} \mathrm{sensibilidade;}$ Espec $=$ especificidade; VPP = valor preditivo positivo; $V P N=$ valor preditivo negativo.

Tabela 3. Área sob a curva ROC, pontos de corte propostos e respectivos valores preditivos para variáveis antropométricas e bioquímicas do sexo masculino.

\begin{tabular}{|c|c|c|c|c|c|c|c|c|}
\hline & Bioquímica & Área (IC95\%) & Pto & $\mathbf{P}$ & Sens & Espec & VPP & VPN \\
\hline \multirow{2}{*}{$\mathrm{IMC}$} & CT & $\begin{array}{c}0,83 \\
(0,74-0,93)\end{array}$ & 19,3 & 64 & 83,3 & 68,1 & 8,9 & 99,1 \\
\hline & LDL-C & $\begin{array}{c}0,87 \\
(0,78-0,95)\end{array}$ & 19,3 & 64 & 83,3 & 67,5 & 8,7 & 99,1 \\
\hline \multirow{2}{*}{ Ccin } & $\mathrm{CT}$ & $\begin{array}{c}0,83 \\
(0,74-0,92)\end{array}$ & 65,7 & 69 & 83,3 & 71,2 & 9,7 & 99,1 \\
\hline & LDL-C & $\begin{array}{c}0,88 \\
(0,81-0,95)\end{array}$ & 65,7 & 69 & 100 & 71,9 & 11,7 & 100 \\
\hline \multirow{2}{*}{ TR } & $\mathrm{CT}$ & $\begin{array}{c}0,86 \\
(0,77-0,96)\end{array}$ & 21,0 & 77 & 83,3 & 78,7 & 12,7 & 99,2 \\
\hline & LDL-C & $\begin{array}{c}0,79 \\
(0,62-0,97)\end{array}$ & 21,0 & 77 & 83,3 & 78,7 & 12,7 & 99,2 \\
\hline
\end{tabular}

Área (IC95\%) = área sob a curva ROC e intervalo de confiança; Pto = ponto de corte proposto; P = percentil em que se encontra; Pto; Sens = sensibilidade; Espec = especificidade; VPP = valor preditivo positivo; VPN = valor preditivo negativo. 
sexo feminino) foi o indicado para a predição de tais alterações lipídicas em ambos os gêneros, apresentando sensibilidade, especificidade e VPN ideais. Esse valor de IMC é ligeiramente superior ao proposto por Katzmarzyk (23) para o sexo masculino $\left(19,1 \mathrm{~kg} / \mathrm{m}^{2}\right)$ na predição de fatores de risco agrupados na faixa etária em questão e inferior para o sexo feminino $\left(19,9 \mathrm{~kg} / \mathrm{m}^{2}\right)$.

Mostrou-se inferior, também, aos valores referenciais nacionais e internacionais para identificação de sobrepeso $(24,25)$ para meninas $\left(19,5\right.$ e $20,7 \mathrm{~kg} / \mathrm{m}^{2}$, respectivamente) e meninos (19,7 e $20,5 \mathrm{~kg} / \mathrm{m}^{2}$, respectivamente). Essas comparações são importantes, uma vez que o objetivo do IMC é identificar crianças e jovens com riscos à saúde aumentados para, posteriormente, serem propostas intervenções no estilo de vida desses indivíduos.

Assim, a amostra estudada pode utilizar o valor de IMC $19,3 \mathrm{~kg} / \mathrm{m}^{2}$, inferior ao preconizado nacional e internacionalmente, classificando $60 \%$ a $83,3 \%$ dos indivíduos corretamente na presença (sensibilidade) ou não (especificidade) de alterações lipídicas (TG nas meninas; CT e LDL-C nos meninos), estimando que o indivíduo considerado sadio pelo teste apresente probabilidade superior a $88 \%$ de realmente não ser doente (VPN). Recomenda-se, dessa forma, a utilização da proposta de Conde e Monteiro (24) $\left(19,5 \mathrm{~kg} / \mathrm{m}^{2}\right)$, visto que esta se aproxima do valor sugerido anteriormente $\left(19,3 \mathrm{~kg} / \mathrm{m}^{2}\right)$, havendo forte indicação de risco.

\section{Ccin na predição de dislipidemia}

A Ccin é ferramenta importante para identificar sobrepeso e obesidade em crianças que apresentam risco de desenvolvimento de complicações metabólicas e cardiovasculares (8). Valores e percentis de referências já foram desenvolvidos para crianças do México (26), da Itália (27) e da Espanha (28). No entanto, pontos de corte que sugerem relações com fatores de risco são ainda escassos. Moreno e cols. (29) propuseram o percentil 70 (os valores específicos não foram apresentados) para predição de síndrome metabólica em crianças com idade média de 11 anos, as quais apresentaram sensibilidade e especificidade de $76 \%$ e $81 \%$, respectivamente. Maffeis e cols. (30) observaram que o percentil 90 associa-se a múltiplos fatores de risco em indivíduos com idade entre 3 e 11 anos.

Neste estudo, os pontos de corte sugeridos encontram-se nos percentis $77 \mathrm{~cm}(65,1)$ e $69 \mathrm{~cm}(65,7)$ para o sexo feminino e masculino, respectivamente, contudo, mostraram diagnósticos somente para concentrações aumentadas de TG feminino e CT e LDL-C masculino. As medidas de acurácia (sensibilidade e especificidade) variaram entre $60 \%$ e $100 \%$, apontando que esses valores discriminam com elevada capacidade os indivíduos doentes e sadios, principalmente no sexo masculino, podendo assim ser utilizados como preditores de concentrações indesejáveis de TG feminino e CT e LDL-C masculino.

\section{TR na predição de dislipidemia}

Não foram encontrados estudos que sugiram pontos de corte para a TR na predição de doenças cardiovasculares. No entanto, neste estudo, a variável TR apresentou IC significativo para CT e LDL-C masculino. Para as duas variáveis bioquímicas, o teste (TR) mostrou elevada sensibilidade, especificidade e VPN (83,3\%, 78,7\% e 99,2\%, respectivamente). Demonstrando, assim, que o valor da dobra TR, superior a $21 \mathrm{~mm}$, apresenta capacidade preditiva elevada para CT e LDL-C masculino, porquanto um indivíduo considerado sadio pelo teste apresenta 99\% de chances de realmente não estar doente.

Do exposto anteriormente, verificou-se que o IMC $\left(19,3 \mathrm{~kg} / \mathrm{m}^{2}\right)$ é teste diagnóstico para concentrações elevadas de TG no sexo feminino e CT e LDL-C no sexo masculino. Os pontos de cortes ideais para Ccin são: $65,1 \mathrm{~cm}$ e $65,7 \mathrm{~cm}$ para meninas e meninos, respectivamente, na identificação de TG feminino e CT e LDL-C masculino. O valor de $21 \mathrm{~mm}$ para TR prediz concentrações aumentadas de CT e LDL-C masculino. É importante destacar que, em ambos os sexos, nenhuma das variáveis antropométricas apresentou poder diagnóstico para HDL-C.

Os resultados deste estudo evidenciam a utilidade das medidas antropométricas (IMC, Ccin e TR) na predição de alterações lipídicas na infância, visto que são de simples execução, baixo custo e não-invasivos, sendo importante tanto para a vigilância nutricional quanto para o diagnóstico populacional. Estudos adicionais com populações similares à estudada devem ser feitos para generalização destes valores referenciais.

Conflitos de interesse: Os autores declararam que não há conflito de interesse que possa interferir na imparcialidade do trabalho científico.

\section{REFERÊNCIAS}

1. Santos RD, Maranhão RC, Luz PL, Lima JC, Salgado Filho W Avezum A, et al. III Diretrizes brasileiras sobre dislipidemias e diretrizes de prevenção da aterosclerose do Departamento de 
Aterosclerose da Sociedade Brasileira de Cardiologia. Arq Bras Cardiol. 2001;77 Suppl $3: 1-191$.

2. Santiago LM, Sá O, De Carvalho IM, Rocha MG, Palmeiro L, Mesquita EP, et al. Hipercolesterolemia e factores de risco cardiovascular associados, em crianças e adolescentes. Rev Port Cardiol. 2002;21(3):301-13.

3. Gerber ZR, Zielinski P. Fatores de risco de aterosclerose na infância. Um estudo epidemiológico. Arq Bras Cardiol. 1997;64(4):231-6.

4. Moura EC, De Castro CM, Mellin AS, Bueno D. Perfil lipídico em escolares de Campinas, SP, Brasil. Rev Saude Publica. 2000;34(5):499-505.

5. Reeder BA, Liu L, Horlick L. Selective screening for dyslipidemia in a Canadian population. J Clin Epidemiol. 1996;49:217-22

6. Paccaud F, Schlüter-Fasmeyer V, Wietlisbach V, Bovet P. Dyslipidemia and abdominal obesity: An assessment in three general populations. J Clin Epidemiol. 2000;53:393-400.

7. Diretrizes para cardiologistas sobre excesso de peso e doença cardiovascular dos departamentos de aterosclerose, cardiologia clínica e FUNCOR da Sociedade Brasileira de Cardiologia. Arq Bras Cardiol. 2002;78 Suppl 1:1-14.

8. Hirschle V, Arand C, Calcagno ML, Maccalini G, Jadzinsky M. Can waist circumference identify children with the metabolic syndrome? Arch Pediatr Adolesc Med. 2005;159:740-4.

9. Freedma, DS, Serdula MK, Srinivasan SR, Berenson GS. Relation of circumferences and skinfold thicknesses to lipid and insulin concentrations in children and adolescents: the Bogalusa Heart Study. Am J Clin Nutr. 1999;69:308-17.

10. Janssen I, Katzmarzyk PT, Ross R. Body mass index, waist circumference, and health risk. Evidence in support of current National Institutes of Health Guidelines. Arch Intern Med. 2002;162: 2074-9.

11. Kubena KS, McIntosh WA, Georghiades MB, Landmann WA. Anthropometric and health in the elderly. J Am Diet Assoc. 1991;91:1402-7.

12. Gus M, Moreira LB, Pimentel M, Gleisener ALM, Moraes RS, Fuchs FD. Associação entre diferentes indicadores de obesidade e prevalência de hipertensão arterial. Arq Bras Cardiol. 1998;70(2):111-4.

13. Kaye SA, Folson AR, Sprafka JM, Prineas RJ, Wallace RB. Increased incidence of diabetes mellitus in relation to abdominal adiposity im older women. J Clin Epidemiol. 1991;44:329-34.

14. Kaplan A, et al. Clinical chemistry interpretation and techniques. 4th ed. Baltimore: Williams \& Wilkins; 1995.

15. Alvarez BR, Pavan AL. Alturas e comprimentos. In: Petroski EL, editor. Antropometria: técnicas e padronizações. Porto Alegre: Pallotti; 2003. p. 29-51.

16. Marins MO, Lopes MA. Perímetros. In: Petroski EL, editor. Antropometria: técnicas e padronizações. Porto Alegre: Pallotti; 2003. p. 57-69.

17. Benedetti T, Pinho RA, Ramos VM. Dobras cutâneas. In: Petroski EL, editor. Antropometria: técnicas e padronizações. Porto Alegre: Pallotti; 2003. p. 127-40.
18. Schisterman EF, Faraggi D, Reiser B, Trevisan M. Statistical inference for the area under the receiver operating characteristic curve in the presence of random measurement error. Am J Epidemiol. 2001;154(2):174-9.

19. Medronho RA, Perez MA. Testes diagnósticos. In: Medronho RA. Epidemiologia. São Paulo: Atheneu; 2004.

20. Badruddin SH, Khurshid M, Molla A, Manser WW, Lalani R, Vellani CW. Factors associated with elevated serum cholesterol levels in well-to-do Pakistani scholchildren. J Trop Med Hyg. 1991;94:123-9.

21. Webber LS, Osganian V, Suepker RV, Feldman HA, Stone EJ, Elder JP, et al. Cardiovascular risk factors among third grade children in four regions of the United States. Am J Epidemiol. 1995; 141:428-39.

22. Massad E. A teoria bayesiana no diagnostico medico. In: Massad E, Menezes RX, Silveira PSP, Ortega NRS. Métodos quantitativos em medicina. Barueri, SP: Manole; 2004.

23. Katzmarzyk PT. Waist circumference percentiles for Canadian youth 11-18y of age. Eur J Clin Nutr. 2004;58:1011-5.

24. Conde WL, Monteiro CA. Body mass index cutoff points for evaluation of nutritional status in Brazilian children and adolescents. J Pediatr. 2006;82(4):2666-72.

25. Cole TJ, Bellizzi MC, Flegal KM, Dietz WH. Establishing a standard definition for child overweight and obesity worldwide: international survey. BMJ. 2000;320:1240-3.

26. Gomez-Diaz RA, Martinez-Hernandez AJ, Aguilar-Salinas CA, Violante R, Alarcon ML, Villarruel MJ, et al. Percentile distribution of the waist circumference among Mexican pre-adolescents of a primary school in Mexico City. Diabetes Obes Metab. 2005 Nov;7(6):716-21.

27. Zannolli R, Morgese G. Waist percentiles: a simples test for a atherogenic disease? Acta Paediatric. 1999;85:1368-9.

28. Moreno LA, Fleta J, Mur L, Rodriguez G, Sarria A, Bueno M. Waist circumference values in Spanish children: gender related differences. Eur J Clin Nutr. 1999;53:429-33.

29. Moreno LA , Pineda I, Rodríguez G , Fleta J, Sarría A, Bueno $M$. Waist circumference for the screening of the metabolic syndrome in children. Acta Paediatr. 2002;91:1307-12.

30. Maffeis C, Pietrobelli A, Grezzani A, Provera S, Tato L. Waist circumference and cardiovascular risk factors in prepubertal children. Obes Res. 2001;9:179-87.

Endereço para correspondência:

Cláudia Cruz Lunardi

SMU QRS/RCG Casa 926

70630-200 Brasilia DF

E-mail: claudia_lunardi@yahoo.com.br 